\title{
Modified PEGASIS in WSN to increase Network Lifetime
}

\author{
Meenu \\ M.Tech., CSE Deptt \\ Hindu College of engg. \\ Sonepat, Haryana
}

\author{
Vandana \\ A.P., CSE Deptt \\ Hindu College of engg. \\ Sonepat,Haryana
}

\begin{abstract}
This paper present a routing protocol for the applications of Wireless Sensor Network (WSN). PEGASIS protocol is a chain-based routing scheme.One of the key problems for Wireless Sensor Networks (WSNs) is the design of energyefficient Routing Algorithm, because sensor energy is limited.Earliar pegasis protocol is based on two parameters i.e Distance and Residual energy. In this paper modification is being carried out in decision parameter i.e response which check the response of nearby node before transmitting the data as well as specify the proposed algorithm for the modified pegasis protocol. Main aim is to to increase network lifetime as well as increase the presence of live nodes so that more nodes will remain exist.
\end{abstract}

Keywords-Wireless Sensor Network, PEGASIS, Routing Protocol.

\section{Introduction}

The nodes of wireless sensor networks is defined with limited energy. Wireless sensor node deployed into the network to monitor the physical or environmental condition such as temperature, sound, vibration at different location. The data is transfer over the network each sensor consume some energy in receiving data, sending data. The lifetime of the network depend how much energy spent in each transmission. And how we can extend the lifetime of the nodes in which routers protocols play an important role. Wireless sensor network is a network that consists of hundred of small nodes that are spatially distributed which monitor physical or environmental conditions, such as temperature, pressure, area monitoring, polluting monitoring, agriculture. Main concerns lies in transmission and since wireless communications consume significant amounts of battery power while transmitting and receiving data. So we can classify energy consumption in WSN as useful and wasteful sources. Useful energy consumption can be due to

1. Transmitting/receiving data,

2. Processing query requests, and

3. Forwarding queries/data to neighboring nodes.

Wasteful energy consumption can be due to

1. Idle listening to the media,

2. Retransmitting due to packet collisions.

Therefore we should optimize them so that little energy is spent while receiving and transmitting data. Initially protocols are not adapt according to the requirement So we proposed modified PEGASIS of. Hierarchical (clustering) techniques can aid in reducing useful energy consumption.
In this paper we present General PEGASIS, Modified PEGASIS and Proposed algorithm using one more parameter in making decision ,simulation results and conclusion that is draw from the result.

\section{General Pegasis}

The PEGASIS (Power-Efficient Gathering in Sensor Information Systems) protocol forms a chain of the sensor nodes and the chain is formed using a greedy approach, starting from the node farthest to the sink node. The nearest node is send the data to the neighbour node. This procedure is continued until all the nodes are included in the chain. This approach will distribute the energy load evenly among the sensor nodes in the network. Here before passing the information to the adjacent neighbour data aggregation takes place . so we can classify the process into two steps:

a) Chain Construction

b) Gathering Data

Chain Construction

[2]

build a chain using a greedy algorithm to route the data to the leader of all nodes

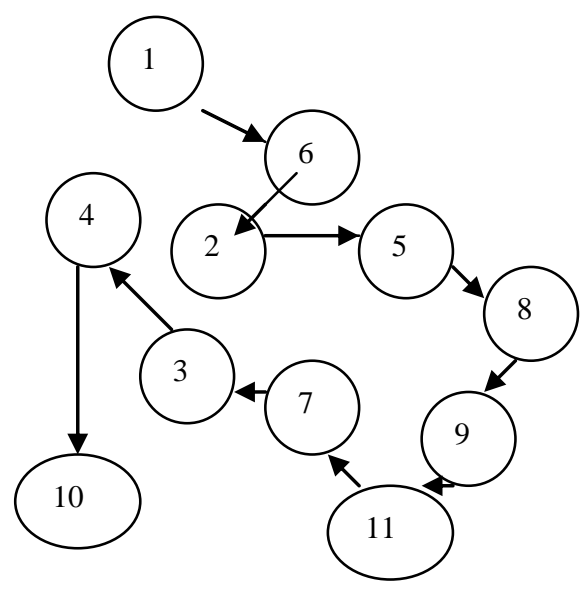

Fig1: Chain is constructed using Greedy Algorithm

In this figure chain is constructed between the nodes and finally node 10 is selected to pass the data to the sink node.

\section{Gathering data}

In this step after leader node is selected and chain is constructed next step is data aggregration and leader node is responsible for forwarding the aggregated data to the sink node. 


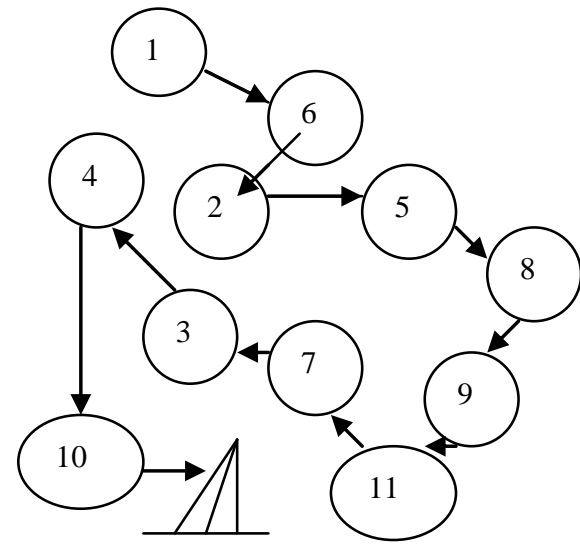

Fig:2 Token Passing approach

\section{Modified Pegasis}

In modified pegasis we are increasing the parameter for making the decision in which route data will be transfer that called Cidel.[5] Cideal is actually defines as the response of the node , means how quickly node is giving response.

\section{CIdel $=$ Throughput - Ceffective .[6]}

It is define as expected and actual response of the node Ceffective is defined as distance*Transmissiondistance*Transmission*overhead

Where overhead value is supposed to be .02

In this we supposed the nodes is suffer form the congestion and congestion is selected randombly Therefore. Throughput is defined throughput $=($ distance + Congestion*distance $) *$ Transmission distance*Transmission*overhead*m

when its value is less then Congestion $<.5$ then $\mathrm{m}$ will be 2 othervise $\mathrm{m}$ will be 3

After chain construction take place, it will check alll the three parameter for making decision and then data is forwarded and in each step data aggregation take place.

\section{PROPOSED ALGORITHM}

\section{Initialize}

Define the Initial and Destination Node called Si and Di

Find the MinDistance and MinDistance Path between Si and Di

Start Communication from Node $\mathrm{Si}$

Find All N Neighbor Nodes of Si called Neighbouri < DistanceVector

\section{Main processing}

For $\mathrm{I}=1$ to $\mathrm{N}$

Find MinDistance,MinEnergy,Cidel for all the Neighbours nodes

If(Energy(Neighbour) $)=$ MinEnergy \&\&Cidel(Neighbour $))<=T$ hreshold \&\& Distance(Neighbour)=MinDistance

Set Neighbour node as Next node

Else if $\quad($ Energy(Neighbour $)=$ MinEnergy and

Distance (Neighbour) $=$ MinDistance

Set Neighbour node as Next node

Else if Distance(Neighbour)=MinDistance

Set Neighbour node as Next node

Else (Energy(Neighbour))=MinEnergy

Set Neighbour node as Next node
Finalize

Set $\mathrm{Si}=$ Selected Neighbour and repeat process for $\mathrm{n}$ rounds

\section{Simulation Results}

To evaluate the performance of the modified Pegasis protocol it has been simulated in 100 node network and the nodes are randomly distributed in a 500 and 500 square.In ths model , a radio dissipates the energy in sending or receiving 1bit: Eelec $=50 \mathrm{~nJ} / \mathrm{bit}$. Energy consumed in every bit data fusion: $E D A=$ $50 \mathrm{pJ} /$ bit Nodes having initial energy is $E=0.5 \mathrm{~J}$.[4] In order to show the performance of the protocol it is simulated using MATLAB. The results are shown under and comparision between them.

4.1Comparison between proposed and existing pegasis protocol

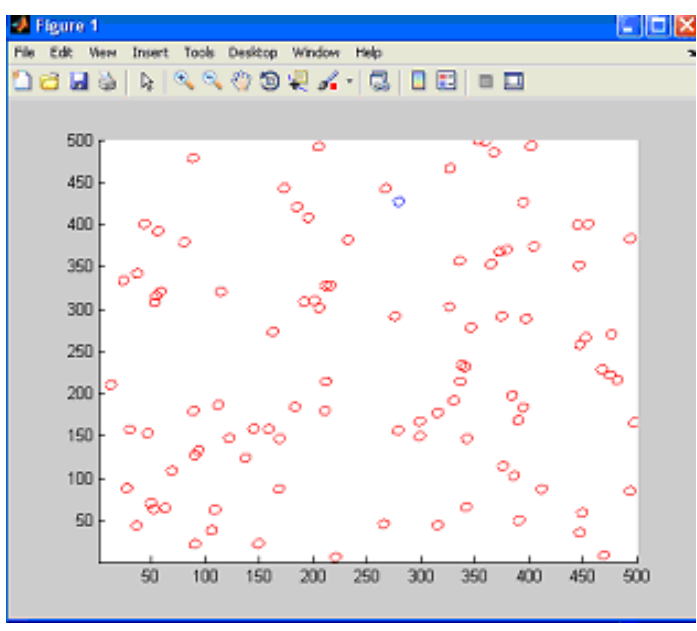

Fig3:Randomly distributed nodes in existing model

In Fig 3 shows the existing PEGASIS protocol in which nodes are randombly distributed

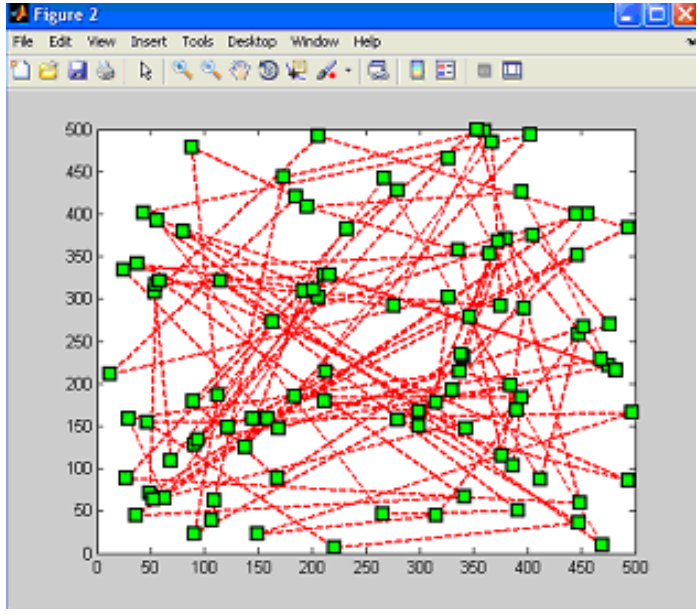

Fig4:Path is constructed in previous Pegasis Model

In later figure path is construsted using existing approach which depends upon only two parameters for its decision.i.e Distance and Residual Energy 


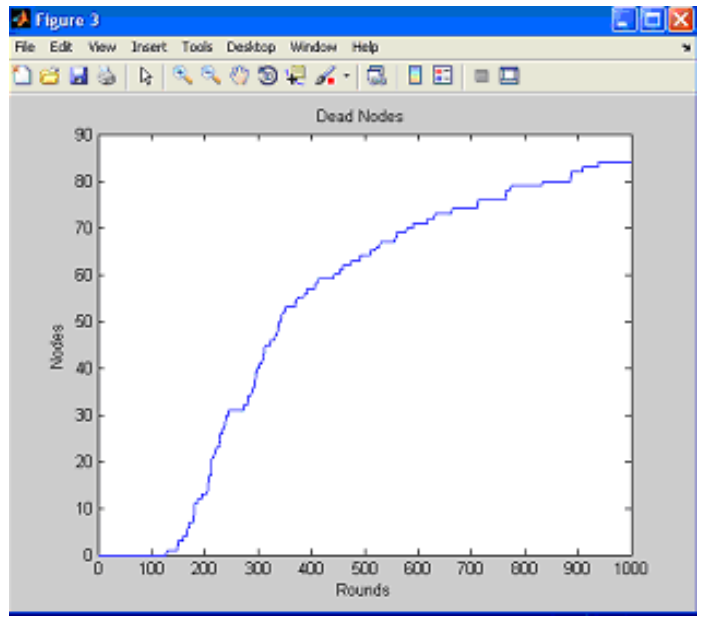

Fig:5 No of dead nodes in Existing Model

In this figure shows after how many rounds the number of nodes become dead.

\subsection{Proposed Model results}

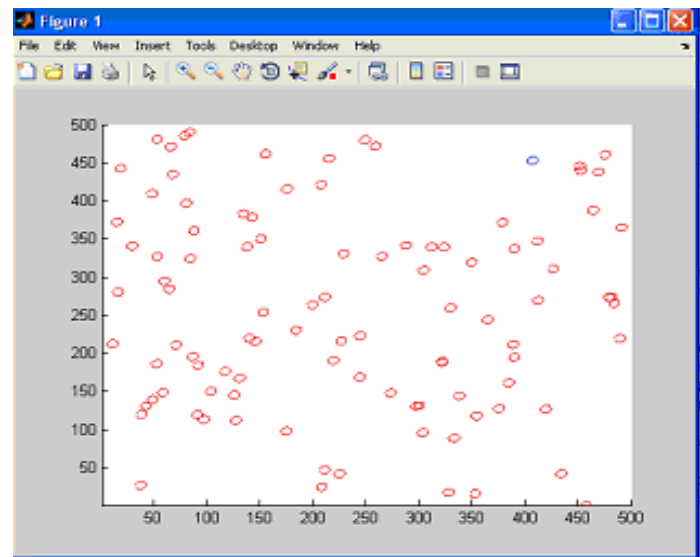

Fig:6Randomly distributed nodes in proposed model

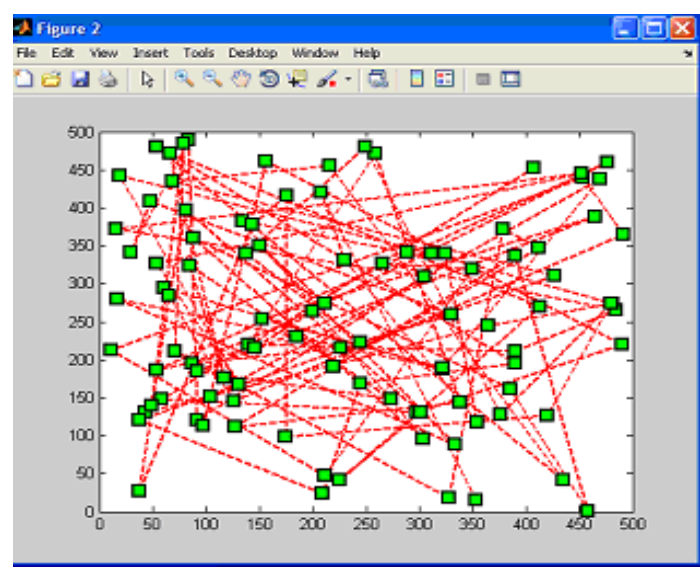

Fig:7Path is formed based on propoesd model

In Fig 6 and Fig 7 nodes are randombly distributed and in later figure path is constructed using given above algorithm In later Fig 8 shows that after how many rounds nodes become dead. The figure shows that number of nodes that become dead is less than the number of nodes in previous model.

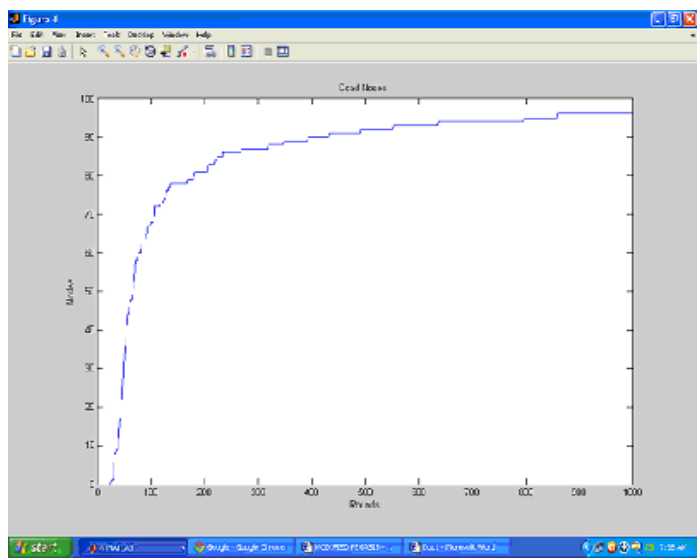

Fig:8No of dead nodes in Modified model

\subsection{Energy Graph between both the Models}

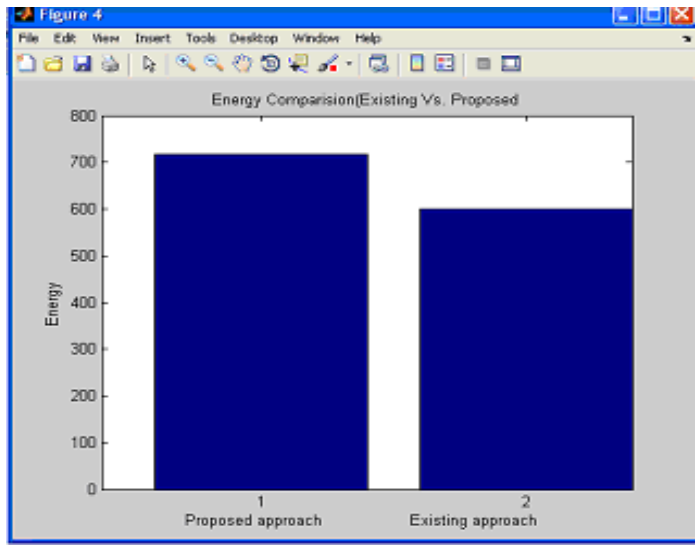

Fig9: Energy Graph between Existing and Proposed Model

In Existing model total energy consumption is 717.4462 and In Proposed model total energy consumption is 603.2271

Different parameters used in model shows are

\begin{tabular}{|l|l|l|}
\hline Parameter & Existing & Proposed \\
\hline Sensor field & $500 \times 500$ & $500 \times 500$ \\
\hline Node Number & 100 & 100 \\
\hline $\begin{array}{l}\text { Energy } \\
\text { consumption }\end{array}$ & 717.4462 & 603.2271 \\
\hline $\begin{array}{l}\text { No of nodes } \\
\text { live after } \\
\text { round 1000 }\end{array}$ & 4 & 84 \\
\hline $\begin{array}{l}\text { No of nodes } \\
\text { dead after } \\
\text { round } 1000\end{array}$ & 96 & 16 \\
\hline
\end{tabular}

TABLE 4.1

Result shows proposed model is better then existing model 


\section{CONCLUSION}

In this paper, we propose an energy efficient PEGASIS routing scheme to increase network lifetime of sensor networks. Our scheme achieves balance of energy dissipation among the nodes and to increase the existence of more nodes in the network. We evaluate performance of our scheme by using simulation on matlab.

\section{REFERENCES}

[1] Ossama Younis, "HEED: A Hybrid, Energy-Efficient, Distributed Clustering Approach for Ad Hoc Sensor Networks" IEEE TRANSACTIONS ON MOBILE COMPUTING, VOL. 3, NO. 4, OCTOBERDECEMBER 2004

[2] Lindsey and C. Raghavendra, "PEGASIS: PowerEfficient Gathering in Sensor Information Systems," in International Conf. on Communications, 2001.
[3] Jamal N. Al-Karaki, Ahmed E. Kamal, "Routing Techniques in Wireless Sensor Networks A survey", IEEE Wireless Communications, Volume 11, Issue 6, Dec. 2004, pp.6-28.

[4] Yongchang Yu, Yichang Song," An Energy-Efficient Chain-Based Routing Protocol in Wireless Sensor Network" 2010 International Conference on Computer Application and System Modeling (ICCASM 2010),IEEE,pp.486-489

[5] An improved PEGASIS Protocolto enhance energy utilization in WSN VOLUME 2 ISSUE 3 May 2012

[6] Shen Gu, Jiajing Wang," An Enhanced Handover Target Cell Selection Algorithm for WiMAX Network", Proceedings of the 15th Asia-Pacific Conference on Communications (APCC 2009)-184 2009 IEEE,pp-774777 